\title{
Quantitative proteomic profiling of ochratoxin A repression in Penicillium nordicum by protective cultures
}

\author{
Josué Delgado $^{\mathrm{a}, *, 1}$, Félix Núñez ${ }^{\mathrm{a}}$, Miguel A. Asensio ${ }^{\mathrm{a}}$, Rebecca A. Owens ${ }^{\mathrm{b}, *}$ \\ ${ }^{a}$ Food Hygiene and Safety, Institute of Meat and Meat Products, University of Extremadura, 10003 Cáceres, Spain \\ ${ }^{\mathrm{b}}$ Department of Biology, Maynooth University, Maynooth, Co. Kildare, Ireland
}

\section{A R T I C L E I N F O}

\section{Keywords:}

Debaryomyces hansenii

Penicillium chrysogenum

Ochratoxin

PgAFP

Protective cultures

Proteomics

\begin{abstract}
A B S T R A C T
Dry-cured meat products are usually contaminated with moulds during ripening. Although fungal development contributes to the desired sensory characteristics, some moulds, such as Penicillium nordicum are able to produce ochratoxin A (OTA) on meat products. Therefore, strategies to prevent OTA contamination in ripened meat products are required. Microorganisms isolated from these meat products can be adequate as biocontrol agents, given that no negative sensory impact is expected. The PgAFP antifungal protein-producer Penicillium chrysogenum (Pc) and Debaryomyces hansenii (Dh) have been shown to successfully inhibit toxigenic moulds. However, scarce information about the mechanism of action of these biocontrol agents on toxigenic mould inhibition is available. Comparative proteomic analysis is a powerful tool to investigate the physiological response of microorganisms to stimuli. Proteomic analysis was carried out on P. nordicum co-cultured with Pc, Dh, PgAFP, and their combinations on a dry-cured ham-based medium. Additionally, OTA production by $P$. nordicum in the different cultures was measured. The individual inoculation of Pc or Dh repressed OTA production by $P$. nordicum by 5 and 3.15 fold, respectively. A total of 2844 unique $P$. nordicum proteins were identified by proteomic analysis. The impact of the biocontrol agents on the proteome of $P$. nordicum was higher for Pc-containing cultures, followed by Dh-containing treatments. PgAFP alone had minimal impact on the proteome of $P$. nordicum. Proteomic analyses indicated Pc repressed $P$. nordicum OTA production through nutrient competition, potentially reducing glucose availability. Data also suggest that Dh and Pc inhibited P. nordicum through cell wall integrity impairment. Both Pc and Dh seem to hamper $P$. nordicum secondary metabolism (SM) as indicated by lower levels of MAP kinases and SM-associated proteins found in the co-inoculated P. nordicum. This work paves the way to use antifungal agents in the most efficient way to prevent OTA formation in meat products.
\end{abstract}

\section{Introduction}

Dry-cured meat products are a significant delicacy in Southern Europe and they have a considerable economic value in countries such as Spain, Portugal and Italy. In particular, dry-cured hams are ripened for up to 24 months following these stages: dry-salting, post-salting and ripening/ageing. Ripening temperature is progressively increased from 3 to $5{ }^{\circ} \mathrm{C}$ to $25-30^{\circ} \mathrm{C}$, across $6-8$ months in "Serrano" type hams or 18-20 months in Iberian type hams, in parallel with a decrease in water activity $\left(a_{w}\right)$ to ensure microbial stability of these products. The sensory quality of these products improves during the ripening period. The particular ecological conditions during ripening, especially at the end, when the temperature is higher, favour surface colonisation by filamentous fungi and yeasts. These fungi can enhance flavour development (Andrade et al., 2009; Martín et al., 2006).

However, some moulds, mainly Penicillium nordicum, are able to produce mycotoxins in these products. Ochratoxin A (OTA) is the most important mycotoxin found at a high rate in dry-cured meat products colonised with a wild fungal population, reaching levels of $30 \mu \mathrm{g} / \mathrm{kg}$ (Markov et al., 2013; Rodríguez et al., 2012). OTA has nephrotoxic, hepatotoxic and immunotoxic properties (Bezerra da Rocha et al., 2014), and has been classified as a possible human carcinogen (Group 2B) by the International Agency for Research on Cancer (Ostry et al., 2016). Some countries such as Italy have established the maximum level for OTA in pork and derived products such as dry-cured ham at $1 \mu \mathrm{g} / \mathrm{kg}$ (Ministero della Sanità, 1999).

Decontamination strategies, such as the addition of adsorbents or chemicals are not efficient and are difficult to use in these products.

\footnotetext{
* Corresponding authors.

E-mail addresses: jdperon@unex.es (J. Delgado), rebecca.owens@mu.ie (R.A. Owens).

${ }^{1}$ Present address: Heart Clinical Unit, Virgen de la Victoria University Clinic Hospital, Instituto de Investigación Biomédica de Málaga-IBIMA, CIBERCV, University of Málaga, Málaga, Spain.
} 
Additionally, OTA is not only found in the outer layer, but penetrates deeper into the products (Rodríguez et al., 2012).

Thus, prevention of OTA contamination is the best way to protect consumer health. The use of microorganisms isolated from dry-cured meat products as protective cultures, could help prevent OTA contamination in these products while also avoiding sensory alterations. The mould Penicillium chrysogenum (Pc), which produces the antifungal protein PgAFP, and the yeast Debaryomyces hansenii (Dh) have proven to be useful for their potential to inhibit OTA-producing moulds (Delgado et al., 2015a; Núñez et al., 2015; Peromingo et al., 2018). The effect of PgAFP on OTA production by $P$. nordicum has not previously been evaluated. Moreover, the combination of these agents against $P$. nordicum on a meat substrate could have a synergistic effect on OTA contamination of these products. In addition, the mechanism of action of these protective agents against ochratoxigenic moulds has not been completely elucidated. Information about the changes provoked in the toxigenic mould in response to protective agents could contribute to a better understanding of the mechanisms involved in OTA production and inhibition.

Quantitative proteomic analysis can be utilized to investigate the physiological response of microorganisms against a particular factor. Indeed, label-free proteomic analyses have been previously used to unveil the mechanism of action of the antifungal protein PgAFP on several moulds under different conditions (Delgado et al., 2017, 2015b, 2015c). These studies yielded information about the modification of mould proteomes upon PgAFP treatment, and illuminated the main proteins and pathways involved in the resistance or sensitivity to this antifungal protein. Thus, proteome analyses would be a very useful tool for revealing $P$. nordicum proteins involved in OTA reduction caused by protective agents.

The objective of this work was to evaluate the effect of Pc, Dh, PgAFP, alone and in combination treatments, on the ability of $P$. nordicum to produce OTA when grown in dry-cured ham-based medium, as well as the comparative quantitative proteomic analysis of $P$. nordicum, to reveal the proteins involved in the reduction of OTA levels.

\section{Materials and methods}

\subsection{Mould strains and culture conditions}

The OTA-producing $P$. nordicum FHSCC Pn15 strain, isolated from dry-cured ham, was evaluated in this work. It was obtained from the Food Hygiene and Safety Culture Collection, University of Extremadura (FHSCC, Cáceres, Spain). The PgAFP-producing P. chrysogenum strain CECT 20922, from the Spanish Type Culture Collection (CECT) and the Debaryomyces hansenii FHSCC $253 \mathrm{H}$ from the Food Hygiene and Safety Culture Collection were used as protective cultures. $P$. nordicum and $P$. chrysogenum were routinely grown on Potato Dextrose Agar (PDA; Scharlab S.L., Spain) for 7 days at $25^{\circ} \mathrm{C}$. Spores from the surface of agar plates were collected using $5 \mathrm{~mL}$ of phosphate saline buffer (PBS) and rubbing the surface with a sterile glass rod. $D$. hansenii was routinely grown in Yeast Extract Sucrose broth (YES; Yeast Extract $20 \mathrm{~g} / \mathrm{L}$, sucrose $125 \mathrm{~g} / \mathrm{L}$, Scharlab) for $72 \mathrm{~h}$ at $200 \mathrm{rpm}$ and $25^{\circ} \mathrm{C}$. The spore/cell suspensions were stored in $10 \%$ glycerol solutions at $-80^{\circ} \mathrm{C}$ and cultures were freshly initiated from stocks for each experiment. Spores and cells were counted using a Thoma chamber.

\subsection{PgAFP purification}

The PgAFP-producing $P$. chrysogenum CECT 20922 was inoculated into Malt Extract Broth (MEB, $20 \mathrm{~g} / \mathrm{L}$ malt extract, $20 \mathrm{~g} / \mathrm{L}$ glucose, and $1 \mathrm{~g} / \mathrm{L}$ peptone; MEB) at $\mathrm{pH} 4.5$, and incubated for 21 days at $25^{\circ} \mathrm{C}$. PgAFP protein was obtained from the cell free medium by fast protein liquid chromatography (FPLC) with a cationic exchange column HiTrap SP HP (Amersham Biosciences, Sweden) and further purified and desalted through a HiLoad 26/60 Superdex 75 gel filtration column for
FPLC (Amersham Biosciences, Sweden) (Acosta et al., 2009).

The concentration of the pooled PgAFP stock solution was measured by the Lowry method (Lowry et al., 1951), sterilised through $0.22 \mu \mathrm{m}$ acetate cellulose filters (Jet Bio-Filtration Co., China), and stored at $-20^{\circ} \mathrm{C}$ until use.

\subsection{Sensitivity of $P$. nordicum FHSCC Pn15 against PgAFP}

In order to test $P$. nordicum FHSCC Pn15 sensitivity against PgAFP, a 96-well microplate with seven two-fold increasing PgAFP concentrations $(1.2-75 \mu \mathrm{g} / \mathrm{mL})$ was prepared using potato dextrose broth (PDB) as culture medium. Additionally a non-treated control was included. Each well was inoculated with $10^{5}$ conidia and incubated at $25{ }^{\circ} \mathrm{C}$ for $96 \mathrm{~h}$, and optical density measured at $595 \mathrm{~nm}$. The assay was run in sextuplicate wells and repeated once.

\subsection{Culture medium preparation}

Dry-cured ham was lyophilised for $24 \mathrm{~h}$ at $5{ }^{\circ} \mathrm{C}$ in a Bulk Tray Dryer with 6-Port Manifold coupled to a FreeZone 6 Liter Console Freeze Dry System (Labconco, USA). Dry-cured ham-based agar was prepared by mixing $30 \mathrm{~g} / \mathrm{L}$ of lyophilised dry-cured ham, $20 \mathrm{~g} / \mathrm{L}$ of Bacto agar (Scharlab), and $70 \mathrm{~g} / \mathrm{L}$ of $\mathrm{NaCl}$, and autoclaving at for $20 \mathrm{~min}$ at $121{ }^{\circ} \mathrm{C}$. This yielded a medium with a similar salt concentration $(7 \%)$ to that found in a dry-cured ham at the end of the ripening stage. PgAFP was added to the medium once cooled to $50^{\circ} \mathrm{C}$, after which it was vigorously shaken and poured into sterile Petri plates. The $\mathrm{a}_{\mathrm{w}}$ values for media measured using water activity meter Novasina Lab Master from Novasina AG (Switzerland), were $0.946 \mathrm{a}_{\mathrm{w}}$ for both non-PgAFP and PgAFP supplemented media.

$P$. nordicum was grown in the presence of protective cultures and/or PgAFP as follows. The protective cultures/treatments consisted of: (a) Dh, (b) Dh + PgAFP, (c) Dh + Pc, (d) Pc and (e) PgAFP alone. A nontreated control was also included and all cultures were prepared in triplicate. Treatments containing PgAFP were prepared by pre-mixing PgAFP $(10 \mu \mathrm{g} / \mathrm{g}$ medium) with agar medium as described above. Protective cultures were applied to the agar surface and spread with a glass rod. All protective cultures were added at a level of $10^{6}$ conidia (Pc) or cells (Dh) per plate. To prevent cross-contamination of fungal tissue between co-cultures, a sterile cellophane sheet (Packaging Limited, UK) was laid over the surface of the plate after spreading the protective cultures. $P$. nordicum $\left(10^{6}\right.$ conidia) was then inoculated onto the cellophane sheet and spread with a glass rod. Therefore, protective cultures and $P$. nordicum were grown separated by the cellophane, as previously described for unveiling fungal interactions at proteome level (Marra et al., 2006). The plates were incubated at $25^{\circ} \mathrm{C}$ for up to 14 days. For proteomic analysis, a portion (50\%) of $P$. nordicum mycelia were harvested from each triplicate on the 5th day, in order to take samples before the mould reached the stationary phase. Mycelia were removed by scraping from the cellophane with a scalpel under sterile conditions and processed as outlined below. The proteome was analysed to reveal the changes at proteome level provoked by each protective culture/treatment. The cultures were further incubated for a total of 14 days to determine OTA levels.

\subsection{OTA extraction and quantification}

\subsubsection{Sampling and OTA extraction procedure}

To evaluate OTA production and secretion into the media by $P$. nordicum, agar samples were collected after 14 days of incubation, once the mould had reached the stationary phase. Dry-cured ham-based agar from $P$. nordicum mixed cultures (a-e) and controls (approximately $1.5 \mathrm{~g}$ ) was placed into $50 \mathrm{~mL}$ conical tubes. The three replicates per treatment were immediately frozen at $-20^{\circ} \mathrm{C}$ and stored until use.

OTA extraction was based on the QuEChERS procedure described by Kamala et al. (2015). Briefly, this method included extraction with 
water and acetonitrile (Scharlab) acidified with $0.1 \%(\mathrm{v} / \mathrm{v})$ acetic acid (Fisher Scientific, USA) prior to phase partitioning using $\mathrm{NaCl}$ (Scharlab) and anhydrous $\mathrm{MgSO}_{4}$ (Scharlab). The mixture was immediately and vigorously shaken by hand and centrifuged for $5 \mathrm{~min}$ at $4^{\circ} \mathrm{C}$ at $3500 \mathrm{~g}$ (Diglicen 21R, Ortoalresa, Spain). Aliquots from the supernatants $\left(0.75 \mathrm{~mL}\right.$, acetonitrile) were stored at $-20^{\circ} \mathrm{C}$ until analysis. Extracts were filtered through a $0.22 \mu \mathrm{m}$ pore size nylon membrane (Jet Bio-Filtration Co. Ltd., China) immediately before analysis.

\subsubsection{OTA quantification by $U H P L C-M S$}

OTA was analysed using a uHPLC system, Dionex UltiMate 3000 (Thermo Scientific, United Kingdom) coupled to an Ion Trap Mass Spectrometer (MS) System, amaZon SL (Bruker Daltonics Inc., Germany). A reversed-phase $\mathrm{C}_{18}$ column was used $(100 \mathrm{~mm} \times 2.1 \mathrm{~mm}$, $2 \mu \mathrm{m}$; Agilent Technologies, USA). The mobile phase consisted of $0.1 \%$ formic acid, $10 \mathrm{mM}$ ammonium formate (solvent A) and acetonitrile (solvent B) and gradient separation was carried out (35 to $98 \% \mathrm{~B}$ ). The injection volume was $10 \mu \mathrm{L}$ and flow rate was set at $0.2 \mathrm{~mL} / \mathrm{min}$. MS detection of OTA was performed using the precursor ion $m / z 404$, and the quantitation ion $\mathrm{m} / \mathrm{z}$ 358. The run time was $15 \mathrm{~min}$, with OTA detected at $8.7 \pm 0.2 \mathrm{~min}$. Signals were processed by Hystar 3.2 software (Bruker Daltonics Inc.)

Standard solutions of OTA were used to generate a calibration curve for uHPLC-MS/MS (1-100 ng/mL) (Sigma Aldrich Co, Germany), with a linear relationship between detector response and amount of OTA standard $\left(R^{2} \geq 0.998\right)$. Limits of detection and quantification (LOD and LOQ) were determined as previously described (Currie, 1999; Long and Winefordner, 1983) and were $1.32 \mu \mathrm{g} / \mathrm{kg}$ and $4.04 \mu \mathrm{g} / \mathrm{kg}$, respectively.

To elucidate whether the combined treatments $(b, c)$ had an antagonistic, additive or synergistic effect on OTA levels, the expected efficacy of this combination was determined by the Abbott formula and the interaction ratio (IR) as described previously (Moreno et al., 2003). IR $<0.5$ denotes an antagonistic effect, IR between 0.5 and 1.5 signify additive interactions and ratios over 1.5 are considered to be synergistic interactions.

\subsection{Proteomic analysis}

\subsubsection{Protein extraction}

$P$. nordicum mycelia were removed by scraping. Mycelia were snap frozen in liquid nitrogen immediately after sampling and stored at $-80^{\circ} \mathrm{C}$ until extraction. Protein extraction was performed as described previously (Delgado et al., 2015c, 2015b). Briefly, following cell lysis, mycelial lysates were centrifuged $(10,000 \times g ; 30 \mathrm{~min})$ to remove cell debris and protein precipitation was carried out on clarified supernatants with trichloroacetic acid/acetone (Carpentier et al., 2005). From these precipitated lysates, label-free quantitative proteomic analysis was performed (Doyle et al., 2018; Sheridan et al., 2016; Sipos et al., 2018).

\subsubsection{Proteome analysis}

The protein precipitated lysates, from three biological replicates per treatment, were resuspended in $8 \mathrm{M}$ urea and treated as previously described (Delgado et al., 2015c, 2015b; Owens et al., 2015). Briefly, following dithiothreitol reduction and iodoacetamide-mediated alkylation, sequencing-grade trypsin (Promega, USA) and ProteaseMAX surfactant (Promega) were added and incubated overnight at $37^{\circ} \mathrm{C}$. Digested samples were desalted prior to analysis using Pierce ${ }^{\mathrm{TM}} \mathrm{C} 18$ Tips (Thermo Scientific). A quantity $(0.75 \mu \mathrm{g})$ was analysed from each digest using a Q-Exactive mass spectrometer coupled to a Dionex Ultimate 3000 RSLCnano (Thermo Scientific). LC gradients ran from 5 to $45 \%$ B (A: $3 \%$ acetonitrile, $0.1 \%$ formic acid (FA), B: $80 \%$ acetonitrile, $0.1 \%$ FA) over $2 \mathrm{~h}$ on an EASY-Spray column, $50 \mathrm{~cm} \times 75 \mu \mathrm{m}$ ID, PepMap RSLC C18, $2 \mu \mathrm{m}$ (Thermo Scientific), and data was collected using a Top15 method for MS/MS scans (Delgado et al., 2017; Dolan et al., 2014). Comparative proteome abundance and data analysis was performed using MaxQuant software (Version 1.6.0.13; www. maxquant.org/downloads.htm) (Cox and Mann, 2008), with Perseus (Version 1.6.0.7) used to organize the data and perform statistical analysis. Carbamidomethylation of cysteines was set as a fixed modification, while oxidation of methionines and acetylation of N-terminals were set as variable modifications. Database searching was performed against a $P$. nordicum protein database (downloaded October 2017, www.uniprot.org). The maximum peptide/protein false discovery rates (FDR) were set to $1 \%$ based on comparison to a reverse database. The LFQ algorithm was used to generate normalized spectral intensities and infer relative protein abundance (Luber et al., 2010). Proteins that matched to a contaminant database or the reverse database were removed, and proteins were only retained in final analysis if detected in at least two replicates from at least one treatment. Quantitative analysis was performed using a $t$-test to compare treatments to the control. Only proteins with a fold change $\geq 2(p<0.05)$ were included in the quantitative results (Delgado et al., 2017, 2015c; Dolan et al., 2014). Qualitative analysis was also performed to detect proteins that were found in at least two replicates of a particular treatment but undetectable in the comparison sample. Functional analysis of identified proteins was carried out using BLAST2GO software, with associated enrichment analysis using gene ontology (GO) terms and a Fisher's exact test (Conesa et al., 2005; Gotz et al., 2008). GO terms that were significantly enriched among the altered proteome compared to the total $P$. nordicum reference proteome, were identified $(p<0.05)$.

\subsubsection{PgAFP detection in microbial proteomes}

The tryptic peptide FGGECSLK $(m / z$ 693.32) from PgAFP was evaluated in the proteome of the PgAFP-producer $P$. chrysogenum, and also in $P$. nordicum treated with PgAFP, by a parallel monitoring reaction (PRM) in a Q-Exactive Plus mass spectrometer coupled to a Dionex RSLCnano (Thermo Scientific). LC gradients ran from 8 to $21 \%$ B (A: $0.1 \%$ FA, B: $100 \%$ acetonitrile, $0.1 \% \mathrm{FA}$ ) over $30 \mathrm{~min}$, (Thermo Scientific). A total amount of $0.5 \mu \mathrm{g}$ was injected to detect the presence of the antifungal protein PgAFP. The standard curve made with this and other pure peptides from a PgAFP digest $\left(0.1-3.5 * 10^{-6} \mu \mathrm{g}\right)$ revealed a linear response between the sample analysed and the detector response $\left(\mathrm{R}^{2}>0.99\right)$. Every sample was evaluated by PRM to detect PgAFP presence in fungal lysates.

\subsection{Statistical analysis}

Statistical analysis for OTA production was performed using the SPSS v.24.0 software. Data sets were tested for normality using the Shapiro-Wilk test and failed the normality test. Then, non-parametric data analysis was performed using the Kruskal-Wallis rank sum test. Subsequently, the Mann-Whitney $U$ test was applied to compare the median values obtained. The statistical significance was set at $p \leq 0.05$.

\section{Results}

\subsection{Sensitivity of $P$. nordicum to PgAFP}

$P$. nordicum growth was not inhibited by PgAFP, even at the maximum concentration tested $(75 \mu \mathrm{g} / \mathrm{mL}$, data not shown). Previous proteomic analyses of the effects of PgAFP on a resistant mould have been carried out using a concentration of $10 \mu \mathrm{g} / \mathrm{mL}$ PgAFP (Delgado et al., 2015b). Thus a comparable concentration of $10 \mu \mathrm{g}$ PAFP/g dry-cured ham-based agar medium was chosen as the appropriate concentration for testing the response of this toxigenic mould.

\subsection{OTA production}

OTA production by $P$. nordicum in dry-cured ham-based medium was evaluated by uHPLC, with $5 \mathrm{ng} / \mathrm{g}$ OTA detected in non-treated control cultures (Fig. 1). Addition of protective cultures of either $P$. 


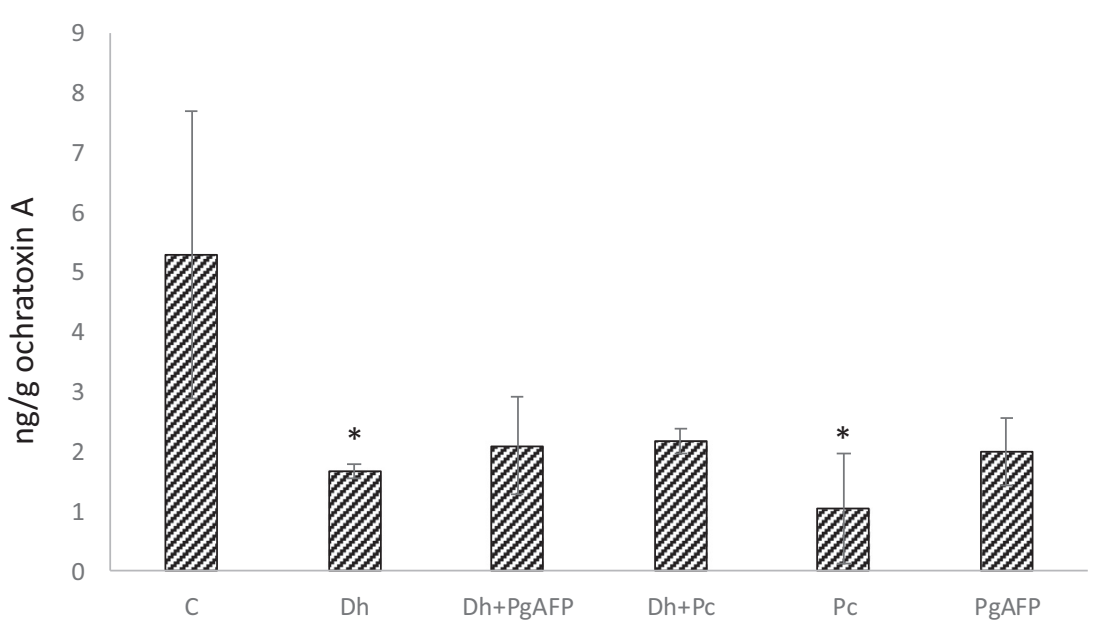

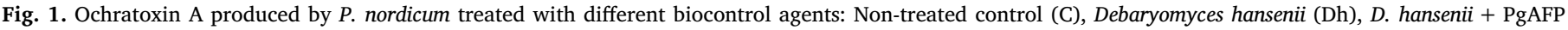
$(\mathrm{Dh}+\mathrm{PgAFP})$, D. hansenii + Penicillium chrysogenum $(\mathrm{Dh}+\mathrm{Pc})$, . chrysogenum $(\mathrm{Pc})$, and PgAFP. * denotes differences $(p \leq 0.05)$ with respect to the control.

chrysogenum or $D$. hansenii significantly reduced the concentration of OTA produced by 5 and 3.15 fold, respectively $(p<0.05)$. While all treatments lead to a reduction in OTA levels compared to the control, this did not reach significance for combined treatments of Dh $+\mathrm{Pc}$, Dh + PgAFP or PgAFP. PgAFP addition with Dh did not produce a significant change in OTA levels, compared to the Dh only treatment $(p>0.05)$. Furthermore, noting the IR yielded by the Abbot formula, the effect of the combination treatments cannot be considered as antagonistic, given the IR $>0.5$ obtained $(0.69$ and 0.9 for Dh + PgAFP and $\mathrm{Dh}+\mathrm{Pc}$ respectively) (Supplementary Table 1 ).

\subsection{Proteome analysis}

In total, 2844 unique $P$. nordicum proteins were identified by proteomic analysis. Quantitative $(p<0.05$; fold change $\geq 2)$ and qualitative (uniquely detected in either condition) changes in protein levels were identified from each treatment (a-e) compared to the control (Table 1; Supplementary Table 2). P. chrysogenum had the highest impact on the $P$. nordicum proteome: combining the qualitative and quantitative changes, 255 proteins were elevated and 700 proteins decreased in abundance in $P$. nordicum following treatment with $P$. chrysogenum alone. In addition, combined treatment of $P$. nordicum with both $P$. chrysogenum and $D$. hansenii led to a total increase in the abundance of 287 proteins, with 679 proteins detected at lower abundance. Hierarchical clustering revealed Pc-containing treatments clustered together, and demonstrated the largest alteration to the $P$. nordicum proteome (Pearson's correlation $(r)$ compared to the control: 0.91-0.92) (Fig. 2). D. hansenii also induced changes in the $P$. nordicum proteome, with 324 proteins increased and 163 proteins decreased in abundance in response to co-cultivation. In contrast, the antifungal protein PgAFP had a very limited effect on the proteome of this ochratoxigenic mould, either alone $(r=0.99)$ or used in combination with $D$. hansenii ( $r=0.98$, compared to Dh treatment alone).

Functional enrichment analysis (BLAST2GO) further confirmed distinct functional profiles in $P$. nordicum proteome alterations for the different treatments (Supplementary Table 3). Cell wall associated proteins, glycosyl hydrolases and proteins involved in secondary metabolism were all altered in abundance in the presence of protective cultures, in addition to reduced levels of ATP and GTP-binding proteins and ribosomal subunits. Among the proteins with elevated abundance following each of the treatments, only one term, extracellular region, was significantly enriched across all treatments $(p<0.05)$. In all treatments, apart from Dh + PgAFP, there was also a significant increase in proteins with transcription regulator activity $(p<0.02)$, including 13 proteins elevated in response to the dual $\mathrm{Dh}+\mathrm{Pc}$ co-culture
(Supplementary Table 3). Among the treatments that included $P$. chrysogenum or PgAFP alone, there was significant increases in proteins associated with carbohydrate metabolic processes $(p<0.005)$, while these were not significantly represented in the $D$. hansenii-responsive proteins. These carbohydrate metabolising proteins were enriched for hydrolases targeting O-glycosyl compounds, with 22, 26 and 7 such proteins increased in abundance in Pc, Dh + Pc and PgAFP treatments, respectively, compared to the control (Supplementary Table 3). Functional enrichment analysis of the proteins that decreased in abundance in the treatments (a-e) compared to the control, revealed proteins associated with the lipid biosynthetic process, protein acylation and cytoskeletal part were significantly represented in all five treatments. Excluding the PgAFP alone treatment, there was a significant decrease in proteins associated with ATP and GTP binding $(p<0.001)$, ribosomal proteins $(p<0.05)$, protein transport $(p<0.01)$, unfolded protein binding $(p<0.01)$, glycosylation $(p<0.05)$, protein serine/threonine kinase activity $(p<0.05)$ and trehalose biosynthetic process $(p<0.05)$ in the remaining four treatments. Proteins associated with RNA binding, vesicle-mediated transport, translational initiation and positive regulation of proteolysis were significantly reduced in abundance in the $P$. chrysogenum containing treatments $(p<0.05)$.

\subsubsection{Cell wall-related proteins}

Among the proteins reduced in abundance in $P$. nordicum, due to the effect of $P$. chrysogenum, were: chitin synthase C, cytoskeleton assembly control protein, five septins (A0A0M8PIA5, A0A0M8NYV2, A0A0M9WI13, Spn2, Spn3), actins (Act1, A0A0M8P359), chitin biosynthesis protein (Chs5) and chitin synthase activator (Chs3), all related to the cell wall integrity (CWI) pathway (Binder et al., 2010; Yoshimi et al., 2016). Several proteins involved in the CWI signaling pathway, including kinases (MpkA, MpkB and Bck1), Rho GTPases and Rho GTPase-regulating proteins were observed to decrease in abundance in response to $\mathrm{Dh}$ and Pc-containing treatments (Table 2). Protein kinase $\mathrm{C}$ (Pkc1), was significantly decreased in response to all protective cultures $(p<0.05)$, although the observed fold change was below the cut-off for analysis (fold change 1.4-1.9).

Interestingly, a chitin binding domain protein and a class III chitinase 1 were detected at lower abundance in $P$. nordicum following treatment with $P$. chrysogenum, alone and in combination with $D$. hansenii. Notably, 1,3-beta-glucanosyltransferase Bgt1 was significantly increased in all Pc-containing treatments compared to the control.

\subsubsection{Secondary metabolism-related proteins}

While proteins associated with OTA production were not detected in 


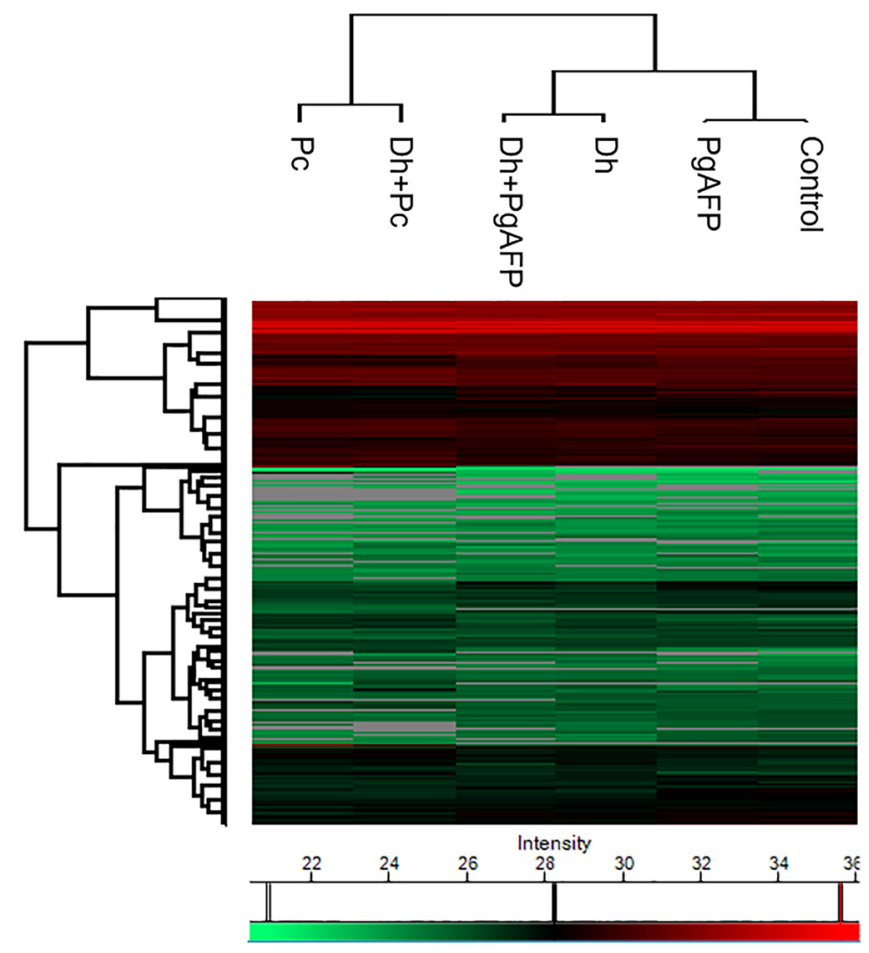

Fig. 2. Heat map showing the hierarchical clustering from label free quantitative data for every $P$. nordicum proteome under different treatments. The mean $\log _{2}$ (intensity) is shown from lowest (green) to highest (red) for each $P$. nordicum protein identified in this study, with grey indicating the protein was not detected in the respective sample. (For interpretation of the references to color in this figure legend, the reader is referred to the web version of this article.)

Table 1

Proteins with altered in abundance in $P$. nordicum proteome, treated with different biocontrol agents.

\begin{tabular}{lcccc}
\hline Control vs. & $\begin{array}{l}\text { ND in } \\
\text { treatment }\end{array}$ & $\begin{array}{l}\text { Down in } \\
\text { treatment }\end{array}$ & $\begin{array}{l}\text { Unique to } \\
\text { treatment }\end{array}$ & $\begin{array}{l}\text { Up in } \\
\text { treatment }\end{array}$ \\
\hline $\mathrm{Dh}$ & 88 & 236 & 53 & 107 \\
$\mathrm{Dh}+\mathrm{PgAFP}$ & 106 & 151 & 54 & 74 \\
$\mathrm{Dh}+\mathrm{Pc}$ & 276 & 403 & 58 & 229 \\
$\mathrm{Pc}$ & 252 & 448 & 75 & 180 \\
PgAFP & 43 & 18 & 22 & 6 \\
\hline
\end{tabular}

ND: not detected in treatment and present in control.

any of the samples tested, a number of other proteins associated with secondary metabolite biosynthesis were altered in abundance in response to protective cultures. Interestingly, a protein with homology to sterigmatocystin 8-O-methyltransferase AflP (BLASTp: E value $9 \mathrm{e}-36$, query coverage $81 \%$, identity $27 \%$ ), associated with aflatoxin production, was significantly decreased in abundance in response to all cocultures, with the exception of the PgAFP treatment. In line with this, a polyketide synthase (PKS; A0A0M9WKE3), with homology to Aspergillus parasiticus AflC (BLASTp: E value 0.0, query coverage $100 \%$, identity $41 \%$ ) showed a similar decrease in abundance upon co-culture with either Dh or Dh + PgAFP. This PKS was also detected at lower abundance in the Pc and Dh + Pc treatments, however no significance could be determined in these cases since the protein was only detected in a single replicate of each treatment. Collectively, these results indicate a reduction in secondary metabolite biosynthetic processes in $P$. nordicum following co-culture with either $D$. hansenii or $P$. chrysogenum, but not PgAFP alone.

\subsubsection{PgAFP detection in microbial proteomes}

Trypsin-mediated digestion of proteins from $P$. nordicum for labelfree proteomic analysis would also potentially allow detection of PgAFP taken up by the treated mould. The tryptic peptide FGGECSLK from PgAFP was not detected in any sample treated with this antifungal protein, nor in the producer $P$. chrysogenum. This finding could mean that $P$. chrysogenum is not producing this antifungal protein under these specific conditions, or that is produced at levels below the limits of detection.

\section{Discussion}

To the best of our knowledge, this is the first proteomic study to evaluate the interaction between protective agents and an ochratoxigenic $P$. nordicum isolated from dry-cured ham, on a meat-based model. This model reflected the results of earlier studies, demonstrating the ability of bioprotective cultures to reduce OTA levels from mycotoxinproducing moulds (Peromingo et al., 2018; Rodríguez et al., 2015; Virgili et al., 2012). Systems-wide comparative proteomics revealed the effect of these combinatorial cultures on $P$. nordicum, identifying changes to carbohydrate metabolism, natural product pathways, transcriptional regulation and the cell wall integrity proteins. The results from these analyses serve to expand our understanding of the interactions between protective and mycotoxigenic fungal species, commonly found on dry-cured meat products.

Combined culture of $P$. nordicum in the presence of either $D$. hansenii or $P$. chrysogenum, lead to a significant reduction in the levels of OTA detected, in a dry-cured ham based medium. While both protective cultures were capable of reducing OTA levels, these were associated with distinct proteomic changes in $P$. nordicum depending on the bioprotective species used. Proteins associated with OTA biosynthesis, including those encoded by $p k s, n p s$ or $p 450-B O 3$ were outside the limits of detection of this analysis, however several groups have carried out gene expression analyses to assess the effect of protective cultures on toxigenic moulds. $D$. hansenii strains have been shown to reduce otanpsPN gene expression in $P$. nordicum, and pks and p450-B03 gene expression in Aspergillus westerdijkiae (Gil-Serna et al., 2011; Peromingo et al., 2018). While the direct relationship between treatments and the OTA biosynthesis pathway in $P$. nordicum could not be elucidated using proteomic analysis, other $P$. nordicum secondary metabolism-related proteins were altered in abundance in mixed cultures. While $P$. nordicum has not been found to produce aflatoxin, decreased levels of proteins with homology to aflatoxin biosynthetic enzymes (AflC, AflP) were observed in response to co-cultures and may indicate differential regulation of secondary metabolism in these conditions. Interestingly, treatment of Aspergillus parasiticus with PgAFP or bioprotective cultures has recently been shown to significantly reduce aflatoxin levels (Delgado et al., 2018), highlighting the links between bioprotective agents and regulation of secondary metabolism.

The proteome of $P$. nordicum revealed a decrease in CWI signaling proteins in response to all tested protective cultures, including the MAP kinases Bck1, MpkA and MpkB. The CWI pathway is activated in response to cell wall damage or remodeling, triggering a signaling cascade, translocation of MpkA to the nucleus and activation of a range of transcription factors (Valiante et al., 2015). Proteins in this pathway are essential to sustain normal hyphal growth, and they control cell wall remodeling through modulation of levels of sugars used in this process (Jain et al., 2011; Müller et al., 2012; Valiante et al., 2009). The cell wall-associated protein 1,3-beta-glucanosyltransferase Bgt1, an ortholog of Saccharomyces cerevisiae Bgl2 (BLASTp: E-value 7e-74, query coverage $91 \%$, identity $42 \%$ ), was elevated in Pc-containing co-cultures. Interestingly, overexpression of $\mathrm{Bgl} 2$ produces a decreased growth rate and cell wall defects associated with a reduction in glucan content (Shimizu et al., 1994). Notably, Bgl2 is negatively regulated by protein kinase C (PKC1) (Shimizu et al., 1994), which was significantly decreased in $P$. nordicum in the presence of all protective cultures 
Table 2

Cell wall integrity-related proteins altered in treated $P$. nordicum.

\begin{tabular}{|c|c|c|c|c|c|c|}
\hline \multirow[t]{2}{*}{ Accession no. (A0A0:) } & \multirow[t]{2}{*}{ Protein name } & \multicolumn{5}{|c|}{ Treatments v control } \\
\hline & & Pc & $\mathrm{Dh}+\mathrm{Pc}$ & $\mathrm{Dh}+\mathrm{PgAFP}$ & Dh & PgAFP \\
\hline M9WET1 & Class III chitinase 1 & 4.2 & 19.0 & & & \\
\hline M8PAR7 & Chitin binding domain & Unique & Unique & & & \\
\hline M8P120 & Chitin synthase $\mathrm{C}$ & ND & ND & & & \\
\hline M9WGB1 & Chitin synthase activator (Chs3) & ND & ND & ND & ND & \\
\hline M9WDJ7 & Chitin biosynthesis (Chs5) & -3.5 & -3.6 & -4.0 & -2.7 & \\
\hline M8P8I8 & Rho GTPase activator (Rgd1) & -4.9 & -4.3 & & & \\
\hline M8PGD6 & Rho GTPase activator (Bem3) & ND & & & & \\
\hline NORZK2 & Rho GTPase activator & ND & & & & \\
\hline M8P0R2 & Rho guanyl nucleotide exchange factor (Rom2) & -2.7 & -2.2 & & & \\
\hline N05005 & GTP-binding RhoA/Rho1 & -2.6 & -2.9 & & & \\
\hline M9WHG6 & RhoGTPase Rho3 & ND & ND & & & \\
\hline M8P3Y3 & MAP kinase (MpkA) & -8.1 & -6.1 & -2.5 & -2.4 & \\
\hline M8P400 & Mitogen-activated kinase (MpkB) & -8.9 & -5.2 & -3.9 & -3.5 & \\
\hline M9W1D6 & MAPKKK (Bck1) & & ND & & & \\
\hline M9WKU6 & Protein kinase C (Pkc1) & -1.7 & -1.8 & -1.9 & -1.4 & \\
\hline M8NYV2 & Septin & -3.6 & -3.7 & -2.1 & & \\
\hline M8PIA5 & Septin & -2.7 & -3.0 & -2.1 & & \\
\hline M9WI13 & Septin & -2.7 & -2.2 & -2.2 & & \\
\hline M8NYW2 & Septin spn2 & -3.2 & -2.6 & -2.8 & & \\
\hline NORYR9 & Septin spn3 & -3.0 & -2.8 & & -2.0 & \\
\hline M8Р359 & Actin & -2.9 & -3.1 & -2.1 & -2.0 & \\
\hline M9WD81 & Actin Act1 & -2.5 & & -2.2 & & \\
\hline M9WF63 & Cytoskeleton assembly control & -3.6 & -3.9 & & & \\
\hline
\end{tabular}

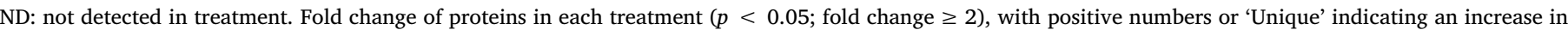
abundance relative to the control. Negative numbers and ND indicate a decrease in protein abundance relative to the control.

( $p<0.05$; fold decrease 1.4-1.87), but not in response to PgAFP (Supplementary Table 2). Collectively, reduced levels of CWI signaling proteins in addition to cell wall biosynthesis enzymes (Chs3, Chs5 and Chitin synthase C) may be a marker of decreased hyphal growth of $P$. nordicum in response to protective culture challenges. Indeed, Hernandez-Montiel et al. (2018) recently outlined cell wall targeting mechanisms utilized by $D$. hansenii during biocontrol of a phytopathogen, leading to reduced hyphal growth and conidial germination of Colletotrichum gloeosporioides. Elevated levels of a conidial hydrophobin Hyp1 in response to Pc-cultures or Dh alone also indicate decreased germination of $P$. nordicum due to protective co-cultures.

In addition to hyphal growth, the CWI signaling pathway has been linked to regulation of secondary metabolism, with the A. fumigatus $\triangle m p k A$ mutant displaying differential expression of a number of genes from secondary metabolite biosynthetic clusters (Müller et al., 2012). This correlates with decreased levels of mycotoxins such as gliotoxin and fumonisin when the CWI pathway is disrupted (Jain et al., 2011; Zhang et al., 2011). D. hansenii reduces fumonisin production, while also partially inhibiting mycelial growth of phytopathogenic fungi (Medina-Córdova et al., 2018). Regulation of secondary metabolism through differential abundance of MAP kinases could contribute to the decreased ochratoxin levels detected following culture of $P$. nordicum with Pc or Dh although this would require further investigation to confirm.

Indications of nutritional competition were evident through changes to the proteome of $P$. nordicum grown in the presence of $P$. chrysogenum or $D$. hansenii. Significant elevation of the glucose-repressible protein Grg1 in all co-culture conditions, reduced levels of hexokinase HxkA in Pc-containing cultures and the absence of the catabolite repressor protein $\mathrm{CreC}$ in the Pc culture all point to a decrease in glucose availability to $P$. nordicum. CreC is part of the carbon catabolite repression (CCR) pathway, which mediates glucose-dependent repression of catabolism of other carbon sources. Interestingly the CCR pathway, has been linked to secondary metabolism, whereby deletion of the creA gene leads to inhibition of aflatoxin production (Fasoyin et al., 2018). Proteins with putative hydrolase activity, including several glucosidases were significantly elevated upon co-culture with $P$. chrysogenum
(Pc, Pc $+\mathrm{Dh}$ ), further supporting nutritional competition and glucose depletion as a mechanism of biocontrol (Supplementary Table 3) (Hernandez-Montiel et al., 2018; Rivas-Garcia et al., 2019). Individual glucosidases are significantly increased in $P$. nordicum with all protective cultures, however the levels were only above the cut-off in Pccontaining cultures. $P$. chrysogenum, therefore, may induce higher nutritional competition compared to $D$. hansenii, leading to more extensive proteome remodeling in Pc-containing co-cultures (Fig. 2).

Considering this, the mechanism of action deployed by $P$. chrysogenum on $P$. nordicum inhibition may be limited to meat products with low sugars content, such as dry-cured ham. In several meat products, such as dry-fermented sausages, different sugars (sucrose, dextrose, lactose, or dextrin) are added at levels c.a. $7 \%$ in the final product (Lizaso et al., 1999). The protective effect of $P$. chrysogenum might be limited in these products, given the additional carbon source readily used by $P$. nordicum. Thus, further studies on carbohydrate enrichedmeat substrates should be carried out to complete the study of the inhibitory effect of $P$. chrysogenum on $P$. nordicum.

Interestingly, the antifungal protein PgAFP had the lowest impact on the P. nordicum proteome. Another PgAFP-resistant mould, P. polonicum, has been reported to modify its proteome to overcome the PgAFP effect (Delgado et al., 2015b). The response of $P$. polonicum to the same PgAFP quantity tested in the present work for $P$. nordicum involved the change in abundance of more proteins than those observed in the present study. The differences in the response can be attributed to the different matrices in which both moulds were grown; $P$. polonicum was cultured in potato dextrose broth and $P$. nordicum in a protein rich drycured ham-based agar, which would hamper the mould-PgAFP interaction. This restricted interaction is underpinned by the fact that the tryptic peptide from PgAFP has not been found in PgAFP-treated mycelia. This antifungal protein was found in $P$. polonicum treated with PgAFP (Delgado et al., 2015b), meaning that the exposure of $P$. nordicum to PgAFP, given the proteome changes observed, was limited in the tested conditions. We should also highlight the fact that PgAFP was not detected even in the producer strain of $P$. chrysogenum, indicating OTA inhibition is linked to nutrient competition and secondary metabolism modulation as discussed above. 
In conclusion, $D$. hansenii and $P$. chrysogenum are effective for OTA control when they act alone against $P$. nordicum in a complex culture medium. This yeast is able to reduce the levels of proteins involved in CWI in $P$. nordicum. P. chrysogenum reduces OTA levels, likely through nutrient competition. D. hansenii and $P$. chrysogenum inoculated together did not lower OTA production possibly due to competition between protective cultures. This work paves the way to use these antifungal agents in the most appropriate way to reduce OTA content, limiting their use in other meat products. Proteomic analysis has revealed a number of potential mechanisms by which these protective agents control OTA production and this will seed further studies to fully elucidate the effect of $P$. chrysogenum and $D$. hansenii on CWI pathway signaling, secondary metabolism and nutritional competition, against the ochratoxigenic $P$. nordicum.

Supplementary data to this article can be found online at https:// doi.org/10.1016/j.ijfoodmicro.2019.108243.

\section{Declaration of Competing Interest}

None.

\section{Acknowledgements}

This work was supported by the Spanish Ministry of Education and Science, Ministry of Economy and Competitiveness, and FEDER (AGL2016-80209-P). Josué Delgado was recipient of a mobility grant from the International Committee on Food Microbiology and Hygiene (ICFMH) to facilitate work carried out in Maynooth University. Labelfree quantitative mass spectrometry facilities were funded by Science Foundation Ireland award to Prof. Sean Doyle (12/RI/2346 (3)).

\section{References}

Acosta, R., Rodríguez-Martín, A., Martín, A., Núñez, F., Asensio, M. a, 2009. Selection of antifungal protein-producing molds from dry-cured meat products. Int. J. Food Microbiol. 135, 39-46. https://doi.org/10.1016/j.ijfoodmicro.2009.07.020.

Andrade, M.J., Córdoba, J.J., Sánchez, B., Casado, E.M., Rodríguez, M., 2009. Evaluation and selection of yeasts isolated from dry-cured Iberian ham by their volatile compound production. Food Chem. 113, 457-463. https://doi.org/10.1016/j.foodchem. 2008.07.080.

Bezerra da Rocha, M.E., Oliveira Freire, F.D.C., Feitosa Maia, F.E., Florindo Guedes, M.I., Rondina, D., 2014. Mycotoxins and their effects on human and animal health. Food Control 36, 159-165. https://doi.org/10.1016/j.foodcont.2013.08.021.

Binder, U., Oberparleiter, C., Meyer, V., Marx, F., 2010. The antifungal protein PAF interferes with PKC/MPK and cAMP/PKA signalling of Aspergillus nidulans. Mol. Microbiol. 75, 294-307. https://doi.org/10.1111/j.1365-2958.2009.06936.x.

Carpentier, S.C., Witters, E., Laukens, K., Deckers, P., Swennen, R., Panis, B., 2005. Preparation of protein extracts from recalcitrant plant tissues: an evaluation of different methods for two-dimensional gel electrophoresis analysis. Proteomics 5, 2497-2507. https://doi.org/10.1002/pmic.200401222.

Conesa, A., Götz, S., García-Gómez, J.M., Terol, J., Talón, M., Robles, M., 2005. Blast2GO: a universal tool for annotation, visualization and analysis in functional genomics research. Bioinformatics 21, 3674-3676. https://doi.org/10.1093/bioinformatics/ bti610.

Cox, J., Mann, M., 2008. MaxQuant enables high peptide identification rates, individualized p.p.b.-range mass accuracies and proteome-wide protein quantification. Nat. Biotechnol. 26, 1367-1372. https://doi.org/10.1038/nbt.1511.

Currie, L.A., 1999. Detection and quantification limits: origins and historical overview. Anal. Chim. Acta 391, 127-134. https://doi.org/10.1016/S0003-2670(99)00105-1.

Delgado, J., Acosta, R., Rodríguez-Martín, A., Bermúdez, E., Núñez, F., Asensio, M.A., 2015a. Growth inhibition and stability of PgAFP from Penicillium chrysogenum against fungi common on dry-ripened meat products. Int. J. Food Microbiol. 205, 23-29. https://doi.org/10.1016/j.ijfoodmicro.2015.03.029.

Delgado, J., Owens, R.A., Doyle, S., Asensio, M.A., Núñez, F., 2015b. Increased chitin biosynthesis contributes to the resistance of Penicillium polonicum against the antifungal protein PgAFP. Appl. Microbiol. Biotechnol. 100, 371-383. https://doi.org/ 10.1007/s00253-015-7020-4.

Delgado, J., Owens, R.A., Doyle, S., Asensio, M.A., Núñez, F., 2015c. Impact of the antifungal protein PgAFP from Penicillium chrysogenum on the protein profile in Aspergillus flavus. Appl. Microbiol. Biotechnol. 99, 8701-8715. https://doi.org/10 1007/s00253-015-6731-x.

Delgado, J., Owens, R.A., Doyle, S., Núñez, F., Asensio, M.A., 2017. Quantitative proteomics reveals new insights into calcium-mediated resistance mechanisms in Aspergillus flavus against the antifungal protein PgAFP in cheese. Food Microbiol. 1-10. https://doi.org/10.1016/j.fm.2017.03.015.
Delgado, J., Rodríguez, A., García, A., Núñez, F., Asensio, M., 2018. Inhibitory effect of PgAFP and protective cultures on Aspergillus parasiticus growth and aflatoxins production on dry-fermented sausage and cheese. Microorganisms 6, 69. https://doi. org/10.3390/microorganisms6030069.

Dolan, S.K., Owens, R.A., O'Keeffe, G., Hammel, S., Fitzpatrick, D.A., Jones, G.W., Doyle, S., 2014. Regulation of nonribosomal peptide synthesis: bis-thiomethylation attenuates gliotoxin biosynthesis in Aspergillus fumigatus. Chem. Biol. 21, 999-1012. https://doi.org/10.1016/j.chembiol.2014.07.006.

Doyle, S., Jones, G.W., Dolan, S.K., 2018. Dysregulated gliotoxin biosynthesis attenuates the production of unrelated biosynthetic gene cluster-encoded metabolites in Aspergillus fumigatus. Fungal Biol. 122, 214-221. https://doi.org/10.1016/J.FUNBIO. 2017.12.007.

Fasoyin, O.E., Wang, B., Qiu, M., Han, X., Chung, K.R., Wang, S., 2018. Carbon catabolite repression gene creA regulates morphology, aflatoxin biosynthesis and virulence in Aspergillus flavus. Fungal Genet. Biol. 115, 41-51. https://doi.org/10.1016/j.fgb. 2018.04.008.

Gil-Serna, J., Patiño, B., Cortés, L., González-Jaén, M.T., Vázquez, C., 2011. Mechanisms involved in reduction of ochratoxin A produced by Aspergillus westerdijkiae using Debaryomyces hansenii CYC 1244. Int. J. Food Microbiol. 151, 113-118. https://doi. org/10.1016/j.ijfoodmicro.2011.08.012.

Gotz, S., Garcia-Gomez, J.M., Terol, J., Williams, T.D., Nagaraj, S.H., Nueda, M.J., Robles, M., Talon, M., Dopazo, J., Conesa, A., 2008. High-throughput functional annotation and data mining with the Blast2GO suite. Nucleic Acids Res. 36, 3420-3435. https:// doi.org/10.1093/nar/gkn176.

Hernandez-Montiel, L.G., Gutierrez-Perez, E.D., Murillo-Amador, B., Vero, S., ChiquitoContreras, R.G., Rincon-Enriquez, G., 2018. Mechanisms employed by Debaryomyces hansenii in biological control of anthracnose disease on papaya fruit. Postharvest Biol. Technol. 139, 31-37. https://doi.org/10.1016/j.postharvbio.2018.01.015.

Jain, R., Valiante, V., Remme, N., Docimo, T., Heinekamp, T., Hertweck, C., Gershenzon, J., Haas, H., Brakhage, A.A., 2011. The MAP kinase MpkA controls cell wall integrity, oxidative stress response, gliotoxin production and iron adaptation in Aspergillus fumigatus. Mol. Microbiol. 82, 39-53. https://doi.org/10.1111/j.1365-2958.2011. 07778.x.

Kamala, A., Ortiz, J., Kimanya, M., Haesaert, G., Donoso, S., Tiisekwa, B., De Meulenaer, B., 2015. Multiple mycotoxin co-occurrence in maize grown in three agro-ecological zones of Tanzania. Food Control 54, 208-215. https://doi.org/10.1016/j.foodcont. 2015.02 .002$.

Lizaso, G., Chasco, J., Beriain, M.J., 1999. Microbiological and biochemical changes during ripening of salchichon, a Spanish dry cured sausage. Food Microbiol. 16, 219-228. https://doi.org/10.1006/fmic.1998.0238.

Long, G.L., Winefordner, J.D., 1983. Limit of detection: a closer look at the IUPAC definition. Anal. Chem. 55, 712A-724A. https://doi.org/10.1021/ac00258a001.

Lowry, O.H., Rosebrough, N.J., Farr, L., Randall, R.J., 1951. Protein measurement with the folin phenol reagent. J. Biol. Chem. 193, 265-275.

Luber, C.A., Cox, J., Lauterbach, H., Fancke, B., Selbach, M., Tschopp, J., Akira, S., Wiegand, M., Hochrein, H., O'Keeffe, M., Mann, M., 2010. Quantitative proteomics reveals subset-specific viral recognition in dendritic cells. Immunity 32, 279-289. https://doi.org/10.1016/j.immuni.2010.01.013.

Markov, K., Pleadin, J., Bevardi, M., Vahčić, N., Sokolić-Mihalak, D., Frece, J., 2013. Natural occurrence of aflatoxin $\mathrm{B}_{1}$, ochratoxin A and citrinin in Croatian fermented meat products. Food Control 34, 312-317. https://doi.org/10.1016/j.foodcont.2013. 05.002 .

Marra, R., Ambrosino, P., Carbone, V., Vinale, F., Woo, S.L., Ruocco, M., Ciliento, R., Lanzuise, S., Ferraioli, S., Soriente, I., Gigante, S., Turrà, D., Fogliano, V., Scala, F., Lorito, M., 2006. Study of the three-way interaction between Trichoderma atroviride, plant and fungal pathogens by using a proteomic approach. Curr. Genet. 50, 307-321. https://doi.org/10.1007/s00294-006-0091-0.

Martín, A., Córdoba, J.J., Aranda, E., Córdoba, M.G., Asensio, M.A., 2006. Contribution of a selected fungal population to the volatile compounds on dry-cured ham. Int. J. Food Microbiol. 110, 8-18. https://doi.org/10.1016/j.ijfoodmicro.2006.01.031.

Medina-Córdova, N., Rosales-Mendoza, S., Hernández-Montiel, L.G., Angulo, C., 2018. The potential use of Debaryomyces hansenii for the biological control of pathogenic fungi in food. Biol. Control 121, 216-222. https://doi.org/10.1016/j.biocontrol. 2018.03.002.

Ministero della Sanità, 1999. Circolare 09.06.1999. In: Sanità, M.D. (Ed.), Circolare 09.06. 1999. Vol. 135 Gazzetta Ufficiale, Repubblica Italiana (11.06.1999). vol. 135.

Moreno, A.B., Del Pozo, A.M., Borja, M., Segundo, B.S., 2003. Activity of the antifungal protein from Aspergillus giganteus against Botrytis cinerea. Phytopathology 93, 1344-1353. https://doi.org/10.1094/PHYTO.2003.93.11.1344.

Müller, S., Baldin, C., Groth, M., Guthke, R., Kniemeyer, O., Brakhage, A.A., Valiante, V., 2012. Comparison of transcriptome technologies in the pathogenic fungus Aspergillus fumigatus reveals novel insights into the genome and MpkA dependent gene expression. BMC Genomics 13, 519. https://doi.org/10.1186/1471-2164-13-519.

Núñez, F., Lara, M.S., Peromingo, B., Delgado, J., Sanchez-Montero, L., J. Andrade, M., 2015. Selection and evaluation of Debaryomyces hansenii isolates as potential bioprotective agents against toxigenic penicillia in dry-fermented sausages. Food Microbiol. 46, 114-120. https://doi.org/10.1016/j.fm.2014.07.019.

Ostry, V., Malir, F., Toman, J., Grosse, Y., 2016. Mycotoxins as human carcinogens-the IARC Monographs classification. Mycotoxin Res. 1-9. https://doi.org/10.1007/ s12550-016-0265-7.

Owens, R.A., O'Keeffe, G., Smith, E.B., Dolan, S.K., Hammel, S., Sheridan, K.J., Fitzpatrick, D.A., Keane, T.M., Jones, G.W., Doyle, S., 2015. Interplay between gliotoxin resistance, secretion, and the methyl/methionine cycle in Aspergillus fumigatus. Eukaryot. Cell 14, 941-957. https://doi.org/10.1128/EC.00055-15.

Peromingo, B., Núñez, F., Rodríguez, A., Alía, A., Andrade, M.J., 2018. Potential of yeasts isolated from dry-cured ham to control ochratoxin A production in meat models. Int. 
J. Food Microbiol. 268, 73-80. https://doi.org/10.1016/j.ijfoodmicro.2018.01.006. Rivas-Garcia, T., Murillo-Amador, B., Nieto-Garibay, A., Rincon-Enriquez, G., ChiquitoContreras, R.G., Hernandez-Montiel, L.G., 2019. Postharvest biology and technology enhanced biocontrol of fruit rot on muskmelon by combination treatment with marine Debaryomyces hansenii and Stenotrophomonas rhizophila and their potential modes of action. Postharvest Biol. Technol. 151, 61-67. https://doi.org/10.1016/j. postharvbio.2019.01.013.

Rodríguez, A., Rodríguez, M., Martín, A., Delgado, J., Córdoba, J.J., 2012. Presence of ochratoxin A on the surface of dry-cured Iberian ham after initial fungal growth in the drying stage. Meat Sci. 92, 728-734. https://doi.org/10.1016/j.meatsci.2012.06. 029.

Rodríguez, A., Bernáldez, V., Rodríguez, M., Andrade, M.J., Núñez, F., Córdoba, J.J., 2015. Effect of selected protective cultures on ochratoxin A accumulation in drycured Iberian ham during its ripening process. LWT Food Sci. Technol. 60, 923-928. https://doi.org/10.1016/j.lwt.2014.09.059.

Sheridan, K.J., Lechner, B.E., Keeffe, G.O., Keller, M.A., Werner, E.R., Lindner, H., Jones, G.W., Haas, H., Doyle, S., 2016. Ergothioneine biosynthesis and functionality in the opportunistic fungal pathogen, Aspergillus fumigatus. Sci. Rep. 6, 1-17. https://doi. org/10.1038/srep35306.

Shimizu, J., Yoda, K., Yamasaki, M., 1994. The hypo-osmolarity-sensitive phenotype of the Saccharomyces cerevisiae hpo2 mutant is due to a mutation in PKC1, which regulates expression of $\beta$-glucanase. Mol. Gen. Genet. 242, 641-648. https://doi.org/10 1007/BF00283417.

Sipos, G., Prasanna, A.N., Walter, M.C., O'Connor, E., Bálint, B., Krizsán, K., Kiss, B., Hess,
J., Varga, T., Slot, J., Riley, R., Bóka, B., Rigling, D., Barry, K., Lee, J., Mihaltcheva, S., LaButti, K., Lipzen, A., Waldron, R., Moloney, N.M., Sperisen, C., Kredics, L., Vágvölgyi, C., Patrignani, A., Fitzpatrick, D., Nagy, I., Doyle, S., Anderson, J.B., Grigoriev, I.V., Güldener, U., Münsterkötter, M., Nagy, L.G., 2018. Author correction: genome expansion and lineage-specific genetic innovations in the forest pathogenic fungi Armillaria. Nat. Ecol. Evol. 1, 1. https://doi.org/10.1038/s41559-018-0469-7.

Valiante, V., Jain, R., Heinekamp, T., Brakhage, A.A., 2009. The MpkA MAP kinase module regulates cell wall integrity signaling and pyomelanin formation in Aspergillus fumigatus. Fungal Genet. Biol. 46, 909-918. https://doi.org/10.1016/j.fgb.2009.08. 005.

Valiante, V., Macheleidt, J., Föge, M., Brakhage, A.A., 2015. The Aspergillus fumigatus cell wall integrity signalling pathway: drug target, compensatory pathways and virulence. Front. Microbiol. 6, 1-12. https://doi.org/10.3389/fmicb.2015.00325.

Virgili, R., Simoncini, N., Toscani, T., Leggieri, M.C., Formenti, S., Battilani, P., 2012. Biocontrol of Penicillium nordicum growth and ochratoxin A production by native yeasts of dry cured ham. pp. 68-82. https://doi.org/10.3390/toxins4020068.

Yoshimi, A., Miyazawa, K., Abe, K., 2016. Cell wall structure and biogenesis in Aspergillus species. Biosci. Biotechnol. Biochem. 8451, 1-12. https://doi.org/10.1080/ 09168451.2016 .1177446$.

Zhang, Y., Choi, Y.E., Zou, X., Xu, J.R., 2011. The FvMK1 mitogen-activated protein kinase gene regulates conidiation, pathogenesis, and fumonisin production in Fusarium verticillioides. Fungal Genet. Biol. 48, 71-79. https://doi.org/10.1016/j.fgb.2010.09. 004 